\title{
(i) firmen\&produkte
}

\section{ARP DATACON \\ Power-Ethernet- Bridge}

Das Stromnetz wird zum LAN mit der Power-Ethernet-Bridge von ARP DATACON. Alles, was der Anwender für eine Datenübertragung von $\mathrm{PC} z \mathrm{u}$ $\mathrm{PC}$ benötigt, ist eine Steckdose mit 230 Volt und eine Ethernet-Karte im Computer, einen Switch oder einen Hub. Bei der Anbindung der externen Power-Ethernet-Bridge erfolgt eine Konvertierung des Stromanschlusses auf einen RI45-Port, der eine Übertragungsrate von $14 \mathrm{Mbit} / \mathrm{s}$ auf Basis des Netzwerkprotokolls TCP/IP liefert.

Der Anschluss erfolgt über Plug\&Play, so dass keine Installationstreiber erforderlich sind. Zur Sicherheit der Daten liefert ARP DATACON eine 56bit-Verschlüsselungssoftware (DES) mit. Damit ein reibungsloser Betrieb des LANs aus der Steckdose gewährleistet ist, sollte alles am selben Elektroverteiler angeschlossen sein. Die Reichweite der IEEE 802.3- und 802.3a-kom-

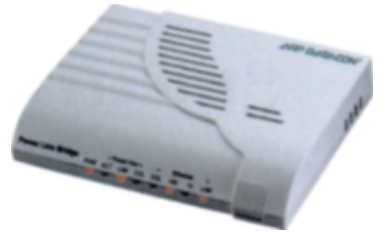

patiblen Geräte liegt bei 30 bis 100 Metern und eignet sich damit ausgezeichnet für kleine Büro- oder Heimnetzwerke.

Informationen: ARP DATACON GmbH, Ing Ewald Wendl, IZ-NÖ Sud, Straße 7, Ob. 58/C8, A-2355 Wr. Neudorf, Telefon +43 (2236) 683 100, Internet: www arp com

\section{SHARP \\ Smart Card-System}

Auf der neuen Smart Card von Sharp, mit 1- MB-Chipkarte, lassen sich Daten verschiedener Ausweise und weiterer Anwendungen integrieren. Der Prozessor der Karte läuft mit bis zu $24 \mathrm{MHz}$, und auch die

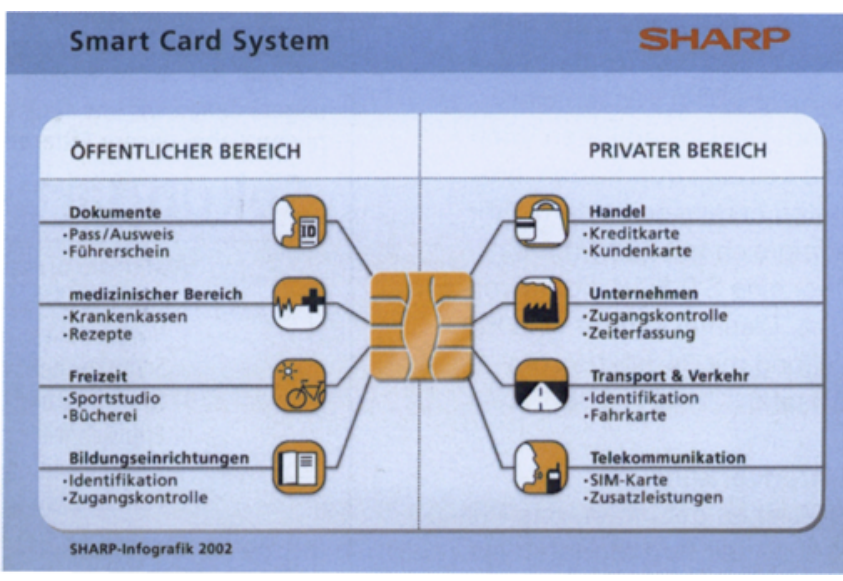

möglichen Datenübertragungsraten liegen beim Vierbis Achtfachen gebräuchlicher Karten. Dadurch passen mehr Daten auf nur eine Karte, und die Einsatzmöglichkeiten vervielfältigen sich.

\section{Anwendungsgebiete}

Ein solcher 1-MB-Chip von Sharp kann gleichzeitig Ausweis, Kantinenkarte, Ticket für den öffentlichen Nahverkehr und Schlüssel sowie das Fahrtenbuch für den Firmenwagen sein. Weitere Anwendungen sind denkbar. Selbst die Speicherung biometrischer Information, also Iris-Abbild oder Fingerabdruck, sind aufgrund der großen Speicherkapazität der Karte möglich. Dadurch ersetzt die Smart Card beispielsweise Personalausweis und Reisepass, Krankenkarte, Studenten, Schüler-, Firmen- oder Bibliotheksausweise. Der Einsatz als Electronic-cash Card oder im privaten Bereich als Zugangscode für Telefon und Computer ist ebenfalls möglich.

Smart Cards sind bereits in über 20 japanischen Städten mit Geld- und Kreditkartenfunktion sowie Ausweisfunktion für öffentliche Einrichtungen im Einsatz. Der ITPassword-Service für den öffentlichen Nahverkehr ist dabei vorbildlich: Im berührunsglosen LeseSchreib-Modus wird die Fahrtgebühr automatisch entfernungsabhängig mit höchster Zuverlässigkeit im mobilen Einsatz abgebucht. Die Benutzer können per mobilem IC-Card-Viewer jederzeit den Stand ihres Guthabens abfragen.

\section{Sicherheit}

Bei so viel sensibler Information spielt Sicherheit eine große Rolle: Daher dienen auf den Karten als Verschlüsselungstechniken unter anderem RSA, Elliptic Curve Cryptography oder ESIGN. Integrierte Firewalls isolieren die geladenen Applikationen voneinander und garantieren ebenfalls Schutz vor Missbrauch.

\section{Vorteile beim Service}

Service und Wartung der Karte ist durch Verwendung der Computersprachen $\mathrm{C}$ und Java stark vereinfacht. Außerdem führt die mehrfache Nutzung nur einer Karte pro $\mathrm{Be}$ nutzer für unterschiedliche Anwendungen zu Kostenvorteilen. Die Kosten können leicht, auch nachträglich, durch Vergabe von Speicherplatz für andere Serviceanbieter umgelegt werden.

Informationen: Sharp Electronics Ges.m.b.H, Handelskai 342, A-1020 Wien, Telefon +43 (1) 72 719-0, In ternet: www.sharpsme.com

\section{Fronlis \\ Photovoltaik- Datenfülle}

Seit kurzem gibt es die FRONIUS IG DatCom, die Datenkommunikation für Photovoltaik(PV)-Anlagen. Damit kann der Photovoltaikbesitzer wichtige Parameter übertragen. speichern und visualisieren. Die DatCom ist flexibel aufgebaut und lässt sich nach den Bedurfnissen des Kunden maßschneidern. Die FRONIUS IG DatCom ist kein Gesamt- gerät, sondern besteht aus verschiedenen Einzelkomponenten, die je nach Wunsch zusammengesetzt werden können und entweder als so genannte Cards oder als Boxes erhältlich sind.

Die Cards sind kleine Platinen, die direkt in den Wechselrichter integriert werden (jedes Gerät verfügt über vier Steckplätze). Boxes sind im Prinzip Cards in einem externen Gehäuse, die mittels RJ 45Stecker ins DatCom-System integriert werden. Dadurch erhält der Anlagenbetreiber einen großen Freiraum in der Platzierung der Komponenten. Alle Wechselrichter und Komponenten dürfen insgesamt bis zu $1.000 \mathrm{~m}$ voneinander entfernt positioniert sein.

\section{Datenlogger Card/Box und} Sensor Card/Box

Die Datenlogger Card/Box ist für die Koordination im ge-
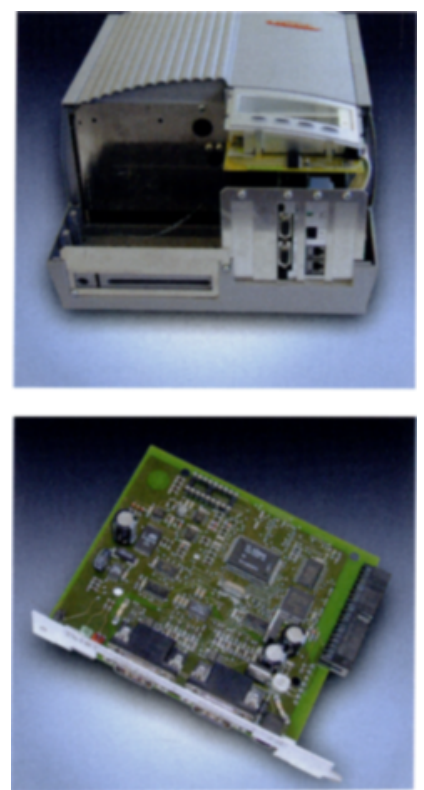

samten DatCom-System sowie für die Speicherung sämtlicher Parameter verantwortlich. Die Datenlogger

Card/Box verwaltet bis zu 100 Wechselrichter und verfügt über eine Schnittstelle zum Anstecken des PCs/Laptops. Mit der Sensor Card/Box erhält der PV-Besitzer durch das Anschließen von Sensoren genaue Umweltdaten wie Einstrahlung, Windgeschwindigkeit oder Modul- und Außentemperatur, die auch gespeichert werden. 